\title{
乌̧
}

\section{Quantum Control of Tightly Competitive Product Channels}

\author{
Matthias Roth, ${ }^{1}$ Laurent Guyon, ${ }^{2}$ Jonathan Roslund, ${ }^{1}$ Véronique Boutou, ${ }^{2}$ Francois Courvoisier, ${ }^{2,3}$ \\ Jean-Pierre Wolf, ${ }^{3}$ and Herschel Rabitz ${ }^{1, *}$ \\ ${ }^{1}$ Department of Chemistry, Princeton University, Princeton, New Jersey 08544, USA \\ ${ }^{2}$ Université Lyon 1, LASIM, UMR CNRS 5579, 43 bd du 11 Novembre 1918, F69622 Villeurbanne Cedex, France \\ ${ }^{3}$ GAP, University of Geneva, 20 rue de l'Ecole de Medecine, CH 1211 Geneva 4, Switzerland
}

(Received 5 February 2009; published 22 June 2009)

\begin{abstract}
Fundamental selectivity limits of quantum control are pushed by introducing laser driven optimal dynamic discrimination to create distinguishing excitations on two nearly identical flavin molecules. Even with modest spectral resources, significant specificity is achieved with optimal pulse shapes, which amplify small molecular differences to create distinct, identifying signals. Rather than being a hindrance, system complexity appears to aid the control process and augments control field capability, which bodes well for implementation of quantum control in a variety of demanding applications.
\end{abstract}

DOI: 10.1103/PhysRevLett.102.253001

PACS numbers: $33.80 .-\mathrm{b}$

Quantum control has proven itself to be quite adept at selectively altering the dynamics of quantum systems across a range of chemical, physical, and biological applications [1]. The systems utilized to show the viability of the concept, however, have traditionally been low resolution with the competitive product channels being wellseparated in state space (e.g., selective electronic state excitation, targeted bond dissociation). Although in principle any well-defined control objective should be attainable [2], the practical capacity for realizing high fidelity control is largely unknown and untested. A good way to assess this matter is through simultaneous control of two erstwhile identical quantum systems, which are then made to differ only slightly by tuning a structural component. Towards this end, the selective identification of target molecules in the presence of structurally and spectroscopically similar background masking agents is a challenge that abounds in multiple areas of science and engineering. The demarcation of tightly competitive excitation channels may prove useful in various areas, including for combatting spectral overlap of multiple cellular agents during in vivo optical imaging, targeted component excitation in solid-state arrays, and transformation of chemically relevant molecules. Recent theoretical analysis yields the general conclusion that quantum systems differing even infinitesimally in structure may be distinguished by means of their dynamics when acted upon by a suitably shaped, ultrafast control field [3]. Such molecular optimal dynamic discrimination (ODD) can in principle achieve dramatic levels of control, and hence provide a valuable testing ground to probe the fundamental selectivity limits of quantum control despite finite laser resources. This Letter reports on experiments that demonstrate control of system selectivity for two structurally similar flavin molecules.

Although specific flavins were chosen for this study, the results should be broadly applicable to control of systems whose static spectra show essentially indistinguishable features associated with the degrees of freedom desired for manipulation. Appropriately, aqueous phase flavin mononucleotide (FMN) and riboflavin (RBF) are nearly identical by the static spectroscopic methods typically utilized for in vivo imaging [4] [Figs. 1(a) and 1(b)]. The flavins' electronic spectroscopy is primarily associated with their common chromophore ( $\pi \rightarrow \pi^{*}$ type transitions localized on the isoalloxazine ring [5]), and is influenced indirectly, and only very slightly, by the chemical moieties $\left(\mathrm{H}\right.$ versus $\left.\mathrm{PO}(\mathrm{OH})_{2}\right)$ on the terminal side chains. As such, absorption spectra for the two flavins are practically indistinguishable throughout the entire visible and far UV, including the UV pump wavelength of $400 \mathrm{~nm}$ (Fig. 1), as

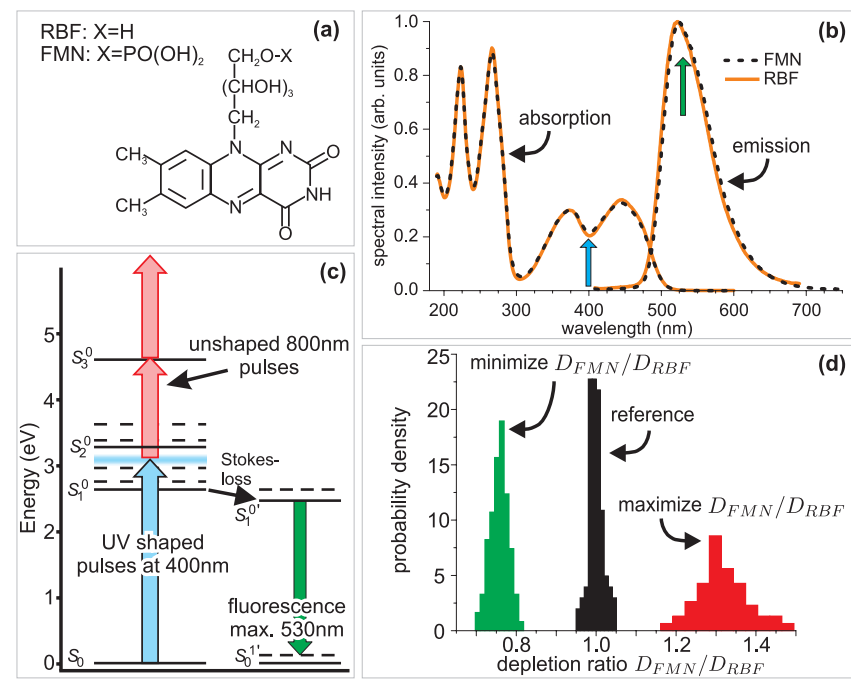

FIG. 1 (color). RBF and FMN are structurally (a) and spectroscopically (b) quite similar. A shaped UV pulse coordinated with a time-delayed IR pulse enables selectivity (c) and is able to both maximize and minimize the fluorescence depletion ratio by $\pm 28 \%$ (d). Arrows at $400 \mathrm{~nm}$ and $530 \mathrm{~nm}$ in (b) locate regions of UV control and collected fluorescence, respectively. 
opposed to only a narrow operating band as in an earlier study [6]. Consequently, achieving any dynamical specificity must rely on the underlying flavin vibronic structure.

This application of ODD may appear to be inordinately complex considering upwards of 100 vibrational degrees of freedom for each flavin, control in a polarizable solvent, and a thermal population of many low-frequency vibrational modes. Yet, rather than acting as a hinderance, this system complexity may be exploited to achieve high excitation specificity even with modest control resources. Importantly, similar circumstances exist for the control of virtually all complex polyatomic molecules, thereby making the findings here of broad significance.

This particular implementation of ODD relies on a two stage control process. First, a specially shaped UV pulse coherently transfers ground state population into a vibrational progression of the flavin electronic states $S_{1}$ or $S_{2}$. Tailoring of the UV spectral phase amplifies minute differences in each system's vibronic structure to create unique dynamical wave packets [Fig. 1(c)]. Regardless of the specific shape of this preparation pulse, the tailored wave packets of both systems eventually evolve to the same final, decohered state when this shaped pulse acts alone. Consequently, any distinction between the two systems is lost in the incoherent, spectrally identical fluorescence signals. However, coordinated application of a second, time-delayed IR pulse on a coherent time scale disrupts the carefully created vibronic excitations and results in an additional excitation that is dependent upon the precise structure, position, and coherence of the tailored wave packets generated by the first pulse. Thus, the action of dual control pulses provides the means to dynamically interrogate the two statically identical systems and thereby produce a discriminating difference in their respective depleted fluorescence signals. The present issue is not to detect the phosphorus moiety, as could be accomplished with NMR, IR, or terahertz spectroscopies, but rather to demonstrate control selectivity limits.

The laser control used in this application consists of an ultraviolet (UV) component at $400 \mathrm{~nm}$ (with an unshaped width of $80 \mathrm{fs}$ ) directly shaped with an acousto-optic modulator (AOM) [7] and an unshaped, 100 fs near infrared (IR) component at $800 \mathrm{~nm}$, which follows the UV component by a time-delay $\tau$ (typically $250-500 \mathrm{fs}$ ). Application of the transform-limited (TL) UV pulse followed by the unshaped IR pulse results in indistinguishable fluorescence depletion signals (Fig. 2) for delay times $\tau$ within the systems' coherence lifetime (Fig. 3 and supporting material [8]), and the lack of any differences for longer times is reflected by the static spectra of Fig. 1. Such indistinguishability indicates equivalent nonlinear absorption cross sections and physical fluorescence depletion mechanisms for RBF and FMN, including the efficiency of ionization, electron transfer, or photoisomerization [9]. The absence of any discernable differences in the relevant multiphoton absorption cross sections necessitates selective control based upon wave packet dynamics. As such,
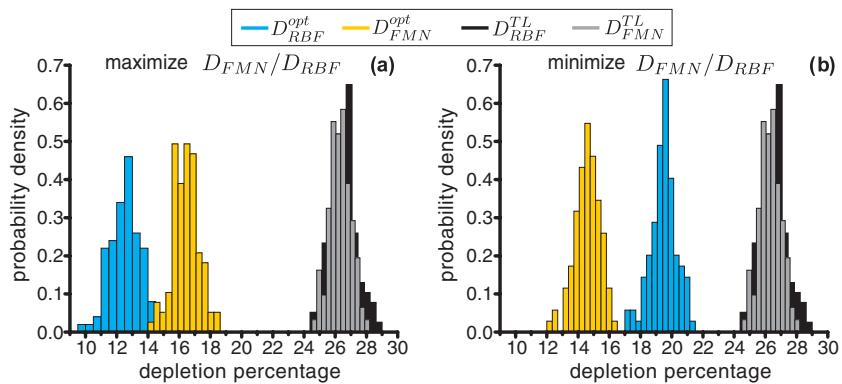

FIG. 2 (color). Absolute RBF and FMN depletion signals for optimized UV pulse shapes at $\tau=500$ fs. Absolute depletions induced by the TL pulse for both RBF (black) and FMN (gray) are statistically equivalent at $\sim 26 \%$. Optimal pulses pull apart the RBF (blue) and FMN (gold) distributions to produce the corresponding depletion ratios shown in Fig. 1(d).

the relative temporal population of the $S_{1}$ electronic state is monitored by measuring the depletion signal $D_{\mathrm{RBF}}(\tau)=$ $\left[F_{\mathrm{RBF}}^{\mathrm{und}}-F_{\mathrm{RBF}}^{d}(\tau)\right] / F_{\mathrm{RBF}}^{\mathrm{und}}$, where $F_{\mathrm{RBF}}^{\mathrm{und}}$ is the undepleted fluorescence signal from the RBF cell (i.e., without application of the IR pulse) and $F_{\mathrm{RBF}}^{d}(\tau)$ is the depleted signal in the presence of the IR pulse delayed by time $\tau$. An analogous expression applies for $D_{\mathrm{FMN}}(\tau)$ recorded with a separate solution of FMN exposed to the same trial pulses.

In order to discover a discriminating UV pulse shape, the RBF and FMN solutions, each with a concentration of $\sim 50 \mu \mathrm{M}$ are located in separate, identical flow cells and exposed to the same trial UV pulse and time-delayed unshaped IR pulse. The nascent, unshaped UV pulse was of intensity $1.8 \times 10^{12} \mathrm{~W} / \mathrm{cm}^{2}$, and the IR pulse was at $2.3 \times 10^{12} \mathrm{~W} / \mathrm{cm}^{2}$. Application of the unshaped IR pulse alone produced no detectable spurious white light and a negligible fluorescence signal; thus, direct two-photon absorption of the interrogating pulse does not play a role in

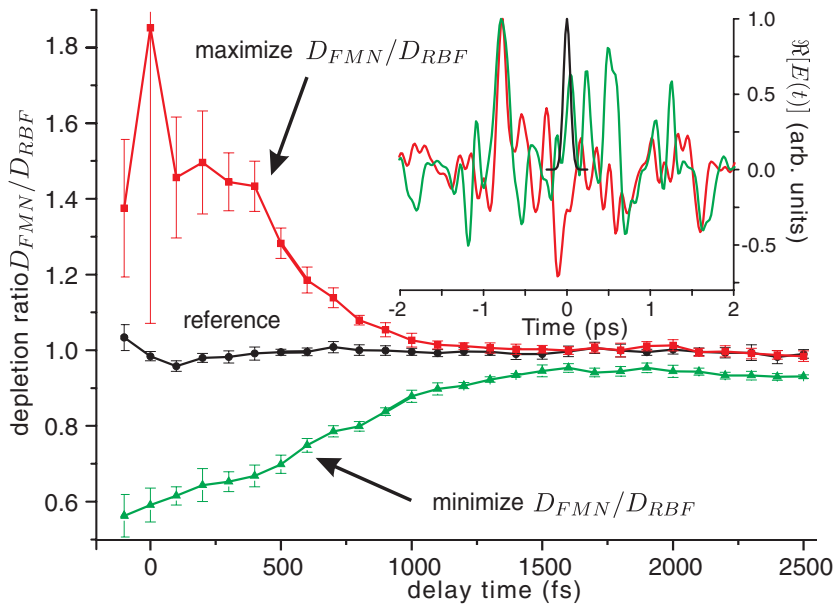

FIG. 3 (color). Flavin ODD as delay $\tau$ is varied between the unshaped IR component and the optimized UV component (optimized at $\tau=500 \mathrm{fs}$ ). Optimal pulses provide good discrimination out to $\tau \sim 1 \mathrm{ps}$. The inset displays the transformlimited pulse and richly structured optimal UV fields. Field colors correspond to depletion signals. 
the initial pump process. The optimal UV pulse is found by closed-loop optimization [10] of the fitness function $J$ for a fixed $\tau: J(\tau)=D_{\mathrm{FMN}}(\tau) / D_{\mathrm{RBF}}(\tau)+0.05 D_{\mathrm{RBF}}(\tau)$, where $D_{\mathrm{FMN}}(\tau) / D_{\mathrm{RBF}}(\tau)=1$ indicates no recognizable discrimination from an unshaped UV pulse. The first term of $J(\tau)$ aims to maximize the depletion ratio of FMN over that of $\mathrm{RBF}$, and the last term prevents the modulation from timeshifting the UV pulse to follow the IR pulse. An analogous expression, with reversal of the roles of RBF and FMN, was used to minimize the depletion ratio of the two systems. This fitness function was extremized at $\tau=500 \mathrm{fs}$ using a 50-dimensional UV spectral phase (maintaining constant UV pulse energy) under the guidance of a genetic algorithm (GA) [10]. Random input phases result in highly unstable depletion signals with very large error bars and no ability to reliably discriminate. Pulses that yield meaningful depletion and discrimination were only discovered under optimization.

For both flavins, application of the IR pulse within $1 \mathrm{ps}$ of the unshaped UV pulse leads to an absolute depletion of $26 \%$ in integrated fluorescence intensity. Under these conditions, the statistically identical depletions for RBF (black histogram) and FMN (gray histogram) in Fig. 2 precludes any discrimination between the two systems. This indistinguishability for all time delays $\tau$ also indicates an equality of the pertinent nonlinear cross sections for both RBF and FMN and an inability to achieve specificity with a simple pump-probe approach. By using closed-loop optimization, however, a particular UV pulse shape was discovered that in tandem with an optimally delayed (unshaped) IR pulse could modulate the relative fluorescence depletion ratio to favor either flavin. Specifically, maximization of $J(\tau)$ yielded a UV/IR pulse pair that induced distinct wave packet dynamics in the two systems so as to create absolute depletion values of $D_{\mathrm{RBF}}=12.6 \%$ (blue histogram) and $D_{\mathrm{FMN}}=16.4 \%$ (gold histogram). The systems are now sufficiently separated dynamically to allow reliable discrimination. Likewise, minimization of $J(\tau)$ discovers a second pulse pair that achieves approximately the same level of discrimination, but reverses the ordering of the $D_{\mathrm{RBF}}$ (blue histogram) and $D_{\mathrm{FMN}}$ (gold histogram) depletions. Overall, the optimized depletion ratio $D_{\mathrm{FMN}} / D_{\mathrm{RBF}}$ could be varied by $\sim \pm 28 \%$ despite the initially indistinguishable linear and nonlinear optical spectra.

Reliable discrimination between the two flavins is now enabled by comparison of each system's optimized depletion ratio $D_{\mathrm{FMN}} / D_{\mathrm{RBF}}$ as seen in Fig. $1(\mathrm{~d})$. These histograms have a well-defined separation of $\sim 56 \%$, defined by $100\left(\mu_{\max }-\mu_{\min }\right)$, where $\mu_{\max }$ and $\mu_{\min }$ are the optimized mean depletion ratios $D_{\mathrm{FMN}} / D_{\mathrm{RBF}}$ of 1.32 and 0.76 , respectively. This separation is equivalently expressed as $12 \sigma$, where $\left(\mu_{\max }-\mu_{\min }\right) / \sigma=12$ and $\sigma=$ $\sqrt{\left(\sigma_{\max }^{2}+\sigma_{\min }^{2}\right) / 2}$. Here $\sigma_{\max }$ and $\sigma_{\min }$ are the standard deviations of the associated probability densities in Fig. 1(d). Hence, adaptive pulse shaping enables a dra- matic degree of separation for the two formerly unresolvable product channels. Achieving this level of discrimination with nearly identical targets in the condensed phase is especially significant, considering that strongly decoherent processes are present.

In order to better understand the controlled dynamics of the ODD process, Fig. 3 displays the discrimination, originally optimized at a delay of $500 \mathrm{fs}$, as the value of $\tau$ is subsequently scanned utilizing the same shaped UV pulses. The fact that significant discrimination could not be attained after a typical solution-phase decoherence time of $\tau \sim 1$ ps suggests the role of coherence in the underlying dynamics. While pulse overlap may be important in the process, it is crucial to note that it is the controlled discrimination that disappears after $\tau \sim 1 \mathrm{ps}$ and not the actual fluorescence depletion, which is observable in both systems for several nanoseconds (not shown). In fact, loss of discrimination is explained by the eventual near return of both RBF and FMN depletions to the transform-limited value of $\sim 26 \%$ after $\tau \sim 1 \mathrm{ps}$. It is expected that phaseonly shaping does not affect the overall population transfer efficiency, and a return is attributed to the ultimate decoherence and relaxation of the transient wave packets in both systems. This long-term return is observed while maximizing the depletion ratio, and the incomplete return of the minimized ratio is due to a gradual degradation of the beamsplitter during the course of the experiment.

Examination of the multiple optimized UV pulse forms that induce these depletions reveals the presence of subpulses with an intricate temporal structure. Because of the high number of normal modes for both flavins, the vibrational state density in $S_{1}$ is quite high. Thus, even for just $3.5 \mathrm{~nm}$ of UV bandwidth, quantum system complexity makes available a large number of active vibrational modes, which allows for detailed wave packet tailoring. Furthermore, the vibrational dynamics of the flavin sidechains are especially critical for the success of ODD, and the sidechain length results in a near continuum of lowfrequency, thermally populated $\left(k T \simeq 210 \mathrm{~cm}^{-1}\right)$ torsional normal modes [11]. Frequency analysis of the temporal subpulse structure shown in Fig. 4 reveals multiple peaks that coincide with these sidechain vibrational modes (see supporting material [8]). Hence, the bandwidth of the UV pump $\left(\Delta \lambda \simeq 220 \mathrm{~cm}^{-1}\right)$ permits impulsive driving of these ground state torsional modes to create interferences in the $S_{1}$ electronic manifold. In this manner, further excitation by the IR pulse to higher lying electronic states appears to be enhanced or diminished depending on the subtle vibrational differences of the two flavins' sidechains.

Repeated application of the closed-loop optimization procedure yielded a family of uniquely shaped UV control pulses that each obtained the same degree of dynamic discrimination (Fig. 4). Although this collection of UV pulses possesses considerable temporal variability, each member is equally successful at dynamically distinguishing the two systems. This finding is consistent with the emerging theory of quantum control landscapes [2], which 
(a) minimize $D_{F M N} / D_{R B F}$
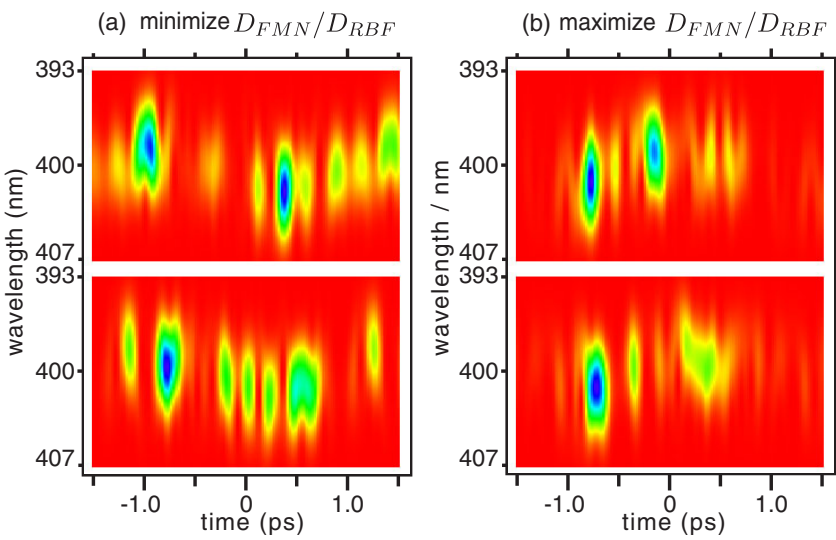

FIG. 4 (color online). Distinct optimal UV pulses that obtain the same degree of discrimination. The short-time Fourier transforms reveal multiple subpulses that commonly show a pattern of frequency downchirp followed by an upchirp for minimization of $D_{\mathrm{FMN}} / D_{\mathrm{RBF}}$ (a) while the solutions for maximization of $D_{\mathrm{FMN}} / D_{\mathrm{RBF}}$ (b) display the opposite chirp pattern. Subtle structure in the UV pulses is crucial, as attempts at creating simplified pulse forms following the observed chirp pattern failed to provide quality discrimination.

predicts the existence of multiple distinct fields providing the same yield [12]. This built-in flexibility demonstrates the ability to achieve successful control by capitalizing upon even limited laser resources.

The assembled set of discriminating laser pulses permits exploiting ODD as a novel means for optical detection in demanding circumstances. To demonstrate such capability, the fluorescence signal from a flavin mixture generated with the $n$th UV-IR control pulse pair is related to the fractional flavin contents by the following equation: $F_{\mathrm{mix}, n}=c_{\mathrm{RBF}} F_{\mathrm{RBF}, n}^{d}+c_{\mathrm{FMN}} F_{\mathrm{FMN}, n}^{d}$, where $F_{\mathrm{RBF}, n}^{d}$ and $F_{\mathrm{FMN}, n}^{d}$ are the depleted fluorescence signals of pure solutions of RBF and FMN, respectively, and $0 \leq c_{\mathrm{RBF}}$, $c_{\mathrm{FMN}} \leq 1$. These standardizing fluorescence signals are recorded for 53.7 and $52.6 \mu \mathrm{M}$ pure, separate solutions of RBF and FMN, respectively, utilizing the previously determined $n$-th optimal pulse. For the purpose of concentration determination, both flavins are combined in a single cell, and the resulting fluorescence $F_{\text {mix }, n}$ from the $n$th optimized pulse is obtained with $1 \mathrm{~min}$ of signal averaging (1 $\mathrm{kHz}$ system operation). Although two distinct ODD pulses could successfully determine the individual fractional contents of the two flavins, an increase in the number of distinct interrogating pulses decreases the extraction error. Accordingly, the retrieval equation is overspecified by measuring the mixture fluorescence at delay times of $\tau=250,375,400,500,600$, and 700 fs for the two optimized pulses that maximize and minimize the depletion ratio $D_{\mathrm{FMN}} / D_{\mathrm{RBF}}$. A typical result from this linear least squares procedure is $c_{\mathrm{RBF}}=0.35 \pm 0.04(18.8 \mu \mathrm{M})$ and $c_{\mathrm{FMN}}=0.68 \pm 0.05(35.8 \mu \mathrm{M})$ when the known fractional contents were $c_{\mathrm{RBF}}=0.33$ and $c_{\mathrm{FMN}}=0.67$. No attempt was made to push the limit of detectibility for this proof-of-concept selectivity control experiment, and these signals were easily measured for concentrations approaching the physiologically significant micromolar level.

In conclusion, although the laser resources exploited for discriminating the nearly identical flavins consisted of a modest $\sim 3.5 \mathrm{~nm}$ of UV bandwidth and $\sim 10 \mathrm{~nm}$ of IR bandwidth, dramatic selectivity was achieved with optimal UV pulses. Whereas the static spectra in Fig. 1(b) appear nearly identical, subtle differences exploited by control are nonetheless profound and allow a flavin discrimination of $12 \sigma$. System complexity (e.g., high vibrational state density, thermal population, solvent-induced line broadening) effectively amplifies the control field capabilities and compensates for the constraint of limited bandwidth, thus making dramatic levels of control possible even in the weak-field limit, a result potentially important for achieving quantum control in numerous demanding applications (see [8]). Additionally, this successful demonstration of ODD specificity bolsters the positive theoretical projections for quantum control and its potential for yielding high selectivity amongst tightly competitive channels in a variety of practical applications [2,3].

The authors acknowledge support from ARO-MURI and NSF grants, and the Swiss NSF.

*hrabitz@princeton.edu

[1] P. Nuernberger, G. Vogt, T. Brixner, and G. Gerber, Phys. Chem. Chem. Phys. 9, 2470 (2007).

[2] T.-S. Ho and H. Rabitz, J. Photochem. Photobiol., A 180, 226 (2006).

[3] B. Li, G. Turinici, V. Ramakrishna, and H. Rabitz, J. Phys. Chem. B 106, 8125 (2002).

[4] P. Heelis, Chem. Soc. Rev. 11, 15 (1982).

[5] E. Sikorska et al., Chem. Phys. 314, 239 (2005).

[6] T. Brixner et al., Nature (London) 414, 57 (2001).

[7] M. Roth, M. Mehendale, A. Bartelt, and H. Rabitz, Appl. Phys. B 80, 441 (2005).

[8] See EPAPS Document No. E-PRLTAO-102-034924 for supplementary material. For more information on EPAPS, see http://www.aip.org/pubservs/epaps.html.

[9] F. Courvoisier et al., J. Photochem. Photobiol., A 180, 300 (2006).

[10] R. S. Judson and H. Rabitz, Phys. Rev. Lett. 68, 1500 (1992).

[11] M. Takahashi, Y. Ishikawa, J. Nishizawa, and H. Ito, Chem. Phys. Lett. 401, 475 (2005).

[12] J. Roslund, M. Roth, and H. Rabitz, Phys. Rev. A 74, 043414 (2006). 